\title{
ACTIONS OF LIE SUPERALGEBRAS ON SEMIPRIME ALGEBRAS WITH CENTRAL INVARIANTS
}

\author{
PIOTR GRZESZCZUK \\ Faculty of Computer Science, Bialystok University of Technology, Wiejska 45A, 15-351 Bialystok, Poland \\ e-mail:piotrgr@pb.edu.pl
}

and MAŁGORZATA HRYNIEWICKA

Institute of Mathematics, University of Bialystok, Akademicka 2, 15-267 Biatystok, Poland e-mail: margitt@math.uwb.edu.pl

\begin{abstract}
Let $R$ be a semiprime algebra over a field $\mathbb{K}$ of characteristic zero acted finitely on by a finite-dimensional Lie superalgebra $L=L_{0} \oplus L_{1}$. It is shown that if $L$ is nilpotent, $\left[L_{0}, L_{1}\right]=0$ and the subalgebra of invariants $R^{L}$ is central, then the action of $L_{0}$ on $R$ is trivial and $R$ satisfies the standard polynomial identity of degree $2 \cdot\left[\sqrt{2^{\operatorname{dim}_{\kappa} L_{1}}}\right]$. Examples of actions of nilpotent Lie superalgebras, with central invariants and with $\left[L_{0}, L_{1}\right] \neq 0$, are constructed.
\end{abstract}

1. Preliminaries. If $R$ is an algebra over a field $\mathbb{K}$ of characteristic $\neq 2$ and $\sigma$ is a $\mathbb{K}$-linear automorphism of $R$ such that $\sigma^{2}=1$, let $D_{0}=$ $\left\{\delta \in \operatorname{End}_{\mathbb{K}}(R) \mid \delta(r s)=\delta(r) s+r \delta(s)\right.$ and $\delta \sigma(r)=\sigma \delta(r)$ for all $\left.r, s \in R\right\}$ and $D_{1}=\{\delta \in$ $\operatorname{End}_{\mathbb{K}}(R) \mid \delta(r s)=\delta(r) s+\sigma(r) \delta(s)$ and $\delta \sigma(r)=-\sigma \delta(r)$ for all $\left.r, s \in R\right\}$. Then $D_{0} \oplus D_{1}$ is a Lie superalgebra and the elements of $D_{0}$ and $D_{1}$ are respectively, derivations and skew derivations of $R$. The superbracket on $D_{0} \oplus D_{1}$ is defined as $\left[\delta_{1}, \delta_{2}\right]=\delta_{1} \delta_{2}-$ $(-1)^{\ddot{j}} \delta_{2} \delta_{1}$, where $\delta_{1} \in D_{i}, \delta_{2} \in D_{j}$ and $i, j \in\{0,1\}$. If $L=L_{0} \oplus L_{1}$ is a Lie superalgebra, we say that $L$ acts on $R$ if there is a homomorphism of Lie superalgebras $\psi: L \rightarrow$ $D_{0} \oplus D_{1}$, where $\psi\left(L_{i}\right) \subseteq D_{i}$, for $i=0,1$. Throughout the paper we will simply assume that $L \subseteq D_{0} \oplus D_{1}$ identifying the elements of $L_{0}$ and $L_{1}$ with their images under $\psi$. It is well known that the homomorphism $\psi$ induces an associative homomorphism from the universal enveloping algebra $U(L)$ to $\operatorname{End}_{\mathbb{}}(R)$ and its image is finite dimensional if and only if the derivations and skew derivations from $L_{0}$ and $L_{1}$ are algebraic. In this case we will say that $L$ acts finitely on $R$. Letting $G$ be the group $\{1, \sigma\}$, we can form the skew group ring $H=U(L) * G$ and $H$ is now a Hopf algebra acting on $R$. When $L$ acts on $R$, we define the subalgebra of invariants $R^{L}$ to be the set $\{r \in R \mid \delta(r)=0$, for all $\delta \in L\}$. Depending upon the context, the symbol [, ] may represent either the superbracket on $L$, or the commutator map $[r, s]=r s-s r$, where $r, s$ belong to an associative algebra. Inductively, we let $L^{1}=L$ and $L^{n+1}=\left[L^{n}, L\right]$ and we say that $L$ is nilpotent if there exists a positive integer $N$ such that $L^{N}=0$. If $R$ (resp. $L$ ) is an associative algebra (resp. Lie superalgebra) we will let $\mathcal{Z}(R)$ (resp. $\mathcal{Z}(L))$ denote its centre. For an element $a \in R$, and automorphism $\sigma$ of $R, \operatorname{ad}_{a}\left(\right.$ resp. $\partial_{a}$ ) stands for the inner derivation (inner $\sigma$-derivation) adjoint to $a$, i.e. $\operatorname{ad}_{a}(x)=a x-x a\left(\partial_{a}(x)=a x-\sigma(x) a\right)$.

2. Main result. The main aim of this paper is to prove the following theorem. 
THEOREM 1. Let a finite-dimensional nilpotent Lie superalgebra $L=L_{0} \oplus L_{1}$ acts finitely on a semiprime $\mathbb{K}$-algebra $R$, where $\mathbb{K}$ is a field of characteristic zero. If $R^{L}$ is central and $\left[L_{1}, L_{0}\right]=0$, then $R$ satisfies the standard polynomial identity of degree $2 \cdot\left[\sqrt{2^{\operatorname{dim}_{\ll} L_{1}}}\right]$.

It generalizes a result from [1] concerning the actions of nilpotent Lie algebras of characteristic zero on semiprime algebras. On the other hand, in [4] it is proved that if a pointed Hopf algebra $H$ acts finitely of dimension $N$ on a semiprime algebra $R$ and the action is such that $L^{H} \neq 0$ for any non-zero $H$-stable left ideal $L$ of $R$ and $R^{H} \subseteq \mathcal{Z}(R)$, then $R$ satisfies PI of degree $2[\sqrt{N}]$. In Theorem 1 we prove for nilpotent Lie superalgebras with $\left[L_{0}, L_{1}\right]=0$, that the dimension of the action of $U(L) * G$ depends only on the dimension of $L_{1}$. The key role will be played by the following easy observation: In characteristic zero the invariants of nilpotent Lie algebras acting on central simple algebras are never proper simple central subalgebras.

LEMMA 2. Let $R$ be a finite-dimensional central simple $\mathbb{F}$-algebra acted on by a nilpotent Lie $\mathbb{F}$-algebra $L$, where $\mathbb{F}$ is a field of characteristic zero. If $R^{L}$ is a central simple $\mathbb{F}$-algebra, then $R=R^{L}$. In this case the action of $L$ on $R$ must be trivial.

Proof. Since $L$ acts by $\mathbb{F}$-linear transformations, any derivation from $L$ is inner. Suppose that the action of $L$ on $R$ is not trivial. Then we can take a non-zero derivation $\delta=\operatorname{ad}_{a} \in \mathcal{Z}(L)$, where $a \in R$. For any $\operatorname{ad}_{b} \in L$ we have $\operatorname{ad}_{[a, b]}=\left[\operatorname{ad}_{a}, \operatorname{ad}_{b}\right]=0$, so $[a, b] \in \mathcal{Z}(R)=\mathbb{F}$. If $[a, b]=\lambda \neq 0$, then $\left[a, \lambda^{-1} b\right]=1$. Note that the elements $a$ and $\lambda^{-1} b$ generate in $R$ a subalgebra isomorphic to the Weyl algebra $\mathbb{A}_{1}(\mathbb{F})$, but it is impossible since $R$ is finite dimensional. Consequently, $[a, b]=0$ for any $\operatorname{ad}_{b} \in L$ and hence $a \in R^{L}$. In particular, $\operatorname{ad}_{a}$ acts trivially on $C_{R}\left(R^{L}\right)$, the centralizer of $R^{L}$ in $R$. On the other hand the subalgebra $R^{L}$ is simple and $\mathcal{Z}\left(R^{L}\right)=\mathbb{F}$, so by Theorem 2 (p. 118) in [5] $R \simeq R^{L} \otimes_{\mathbb{F}} C_{R}\left(R^{L}\right) \simeq R^{L} \cdot C_{R}\left(R^{L}\right)$. Consequently, $R=R^{L} \cdot C_{R}\left(R^{L}\right)$. It implies that $\operatorname{ad}_{a}$ acts trivially on $R$, a contradiction. Therefore the action of $L$ on $R$ is trivial.

Suppose that a finite-dimensional nilpotent Lie superalgebra $L=L_{0} \oplus L_{1}$ acts finitely of dimension $N$ on an algebra $R$. Then by $R_{L_{0}}$ we denote the largest subspace of $R$ on which any derivation from $L_{0}$ acts nilpotently, that is

$$
R_{L_{0}}=\left\{r \in R \mid \delta^{N}(r)=0, \quad \forall \delta \in L_{0}\right\} .
$$

It is clear that $R_{L_{0}}$ is a subalgebra of $R$ and $R_{L_{0}}$ is stable under the automorphism $\sigma$. Furthermore, it is well known that (after eventual extension of the field of scalars) the algebra $R$ is graded (with finite support) by the dual of the Lie algebra $L_{0}$ with $R_{L_{0}}$ as the identity component of the grading. Therefore, if the algebra $R$ is semiprime (semisimple), then $R_{L_{0}}$ is also semiprime (resp. semisimple). In [3] (Lemma 12) it is proved that

LeMma 3. The subalgebra $R_{L_{0}}$ is L-stable. In particular, L acts on $R_{L_{0}}$ by nilpotent transformations.

In the next Proposition we consider the case of action of a nilpotent Lie superalgebra on a finite-dimensional $G$-simple algebra.

Proposition 4. Let a nilpotent Lie superalgebra $L=L_{0} \oplus L_{1}$ acts on a $G$-simple finite-dimensional $\mathbb{K}$-algebra $R$, where $\mathbb{K}$ is a field of characteristic zero. If $R^{L}$ is central and $\left[L_{0}, L_{1}\right]=0$, then $L_{0}=0$. 
Proof. First, we will consider the case when $L$ acts on $R$ by nilpotent transformations, that is, $R=R_{L_{0}}$. Suppose that $L_{0} \neq 0$ and take a non-zero derivation $\delta$ from the centre of $L_{0}$. Since $\left[L_{0}, L_{1}\right]=0$, it is clear that $\delta$ is in the centre of $L$. Let $k>1$ be such that $\delta^{k}(R)=0$ and $V=\delta^{k-1}(R) \neq 0$. Then $V$ is invariant under the action of $L$, and since $L$ acts via nilpotent transformations it is clear that $V^{L}=V \cap R^{L} \subseteq \mathcal{Z}(R)$. On the other hand if $r, s \in R$, then the Leibniz rule gives

$$
0=\delta^{k}\left(\delta^{k-2}(r) s\right)=k \delta^{k-1}(r) \delta^{k-1}(s) .
$$

It means that $\left(V^{L}\right)^{2}=0$, so the centre of $R$ contains nilpotent elements. This is impossible since $R$ is semisimple. The obtained contradiction shows that $L_{0}=0$.

Consider the general case. The above gives us immediately that $R^{L_{0}}=R_{L_{0}}$ and consequently the algebra $R^{L_{0}}$ is semisimple. Thus, any its ideal $I$ is idempotent, i.e. $I^{2}=I$. Note that if $I$ is $G$-stable, then the Leibniz rule, applied to any $\partial \in L_{1}$, gives $\partial(I)=\partial\left(I^{2}\right) \subseteq \partial(I) I+\sigma(I) \partial(I) \subseteq I$. Hence any $G$-stable ideal $I$ of $R^{L_{0}}$ is also $L$-stable and $0 \neq I^{L} \subseteq \mathcal{Z}(R)$. Thus $I$ contains invertible elements. Consequently, $R^{L_{0}}$ is also $G$-simple.

We will split considerations into two cases. First, suppose that the automorphism $\sigma$ is inner, and let $q \in R$ be such that $\sigma(x)=q^{-1} x q$, for $x \in R$. In this case any ideal of $R$ is $\sigma$-stable, so $R$ must be a simple algebra. Moreover it is easy to see that any skew derivation $\partial$ from $L_{1}$ must be inner. Indeed, since $\partial \sigma=-\sigma \partial$, we obtain that

$$
\begin{aligned}
q^{-1} \partial(x) q & =\sigma(\partial(x))=-\partial(\sigma(x))=-\partial\left(q^{-1} x q\right) \\
& =-\partial\left(q^{-1}\right) x q-q^{-1} \partial(x) q-q^{-1} \sigma(x) \partial(q) .
\end{aligned}
$$

Since $q \partial\left(q^{-1}\right)=-\partial(q) q^{-1}$

$$
\partial(x)=-\frac{1}{2} q \partial\left(q^{-1}\right) x-\frac{1}{2} \sigma(x) \partial(q) q^{-1}=\frac{1}{2} \partial(q) q^{-1} x-\sigma(x) \frac{1}{2} \partial(q) q^{-1} .
$$

This immediately gives, that $\partial(x)=b x-\sigma(x) b$, where $b=\frac{1}{2} \partial(q) q^{-1}$. Consequently, any mapping from $L_{0} \cup L_{1}$ is $\mathcal{Z}(R)$-linear. We will show that the algebra $R^{L_{0}}$ is simple and the centres of $R^{L_{0}}$ and $R$ coincide. Since the automorphism $\sigma$ has order $2, q^{2} \in$ $\mathcal{Z}(R)$. Thus for any $\delta=\operatorname{ad}_{a} \in L_{0}$,

$$
\delta(q)=\delta(\sigma(q))=\sigma(\delta(q))=q^{-1}(a q-q a) q=q a-a q=-\delta(q),
$$

so $\delta(q)=0$. This implies that $q \in R^{L_{0}}$, the restriction of $\sigma$ to $R^{L_{0}}$ is inner and hence the algebra $R^{L_{0}}$ is simple. Since the action of $L$ on $R$ is inner, $\mathcal{Z}(R)=\mathcal{Z}(R) \cap R^{L_{0}} \subseteq \mathcal{Z}\left(R^{L_{0}}\right)$. We will show that $\mathcal{Z}\left(R^{L_{0}}\right) \subseteq \mathcal{Z}(R)$. To this end, since $R^{L} \subseteq \mathcal{Z}(R)$, it suffices to show that $\mathcal{Z}\left(R^{L_{0}}\right) \subseteq R^{L_{1}}$. Take any $z \in \mathcal{Z}\left(R^{L_{0}}\right)$, and $\partial=\partial_{b} \in L_{1}$, where $b=\frac{1}{2} \partial(q) q^{-1}$. Notice that $b \in R^{L_{0}}$. Indeed, by assumption $[\delta, \partial]=0$ for any $\delta \in L_{0}$ and by the above $q \in R^{L_{0}}$, so

$$
\delta(b)=\frac{1}{2} \delta\left(\partial(q) q^{-1}\right)=\frac{1}{2} \delta(\partial(q)) q^{-1}+\frac{1}{2} \partial(q) \delta\left(q^{-1}\right)=\frac{1}{2} \partial(\delta(q)) q^{-1}=0 .
$$

It means that $b \in R^{L_{0}}$ and

$$
\partial(z)=b z-\sigma(z) b=b z-z b=0,
$$


so $z \in R^{L_{1}}$. It proves that $\mathcal{Z}\left(R^{L_{0}}\right)=\mathcal{Z}(R)$. By Lemma 2 the action of $L_{0}$ on $R$ must be trivial.

Finally, suppose that the automorphism $\sigma$ is outer. Since $R$ is $G$-simple, the algebra $R$ must be either simple or $R=I \oplus \sigma(I)$ for some minimal ideal $I$. In the first case, by the Skolem-Noether theorem, $\sigma$ is not an identity map on $\mathcal{Z}(R)$. In the second case $\mathcal{Z}(R)=\mathcal{Z}(I) \oplus \sigma(\mathcal{Z}(I))$. Thus in both cases $\sigma$ acts non identically on $\mathcal{Z}(R)$. Now since the centre of $R^{L_{0}}$ contains $\mathcal{Z}(R)$, the restriction of $\sigma$ to $R^{L_{0}}$ is also outer. Consequently, one can choose a non-zero element $c \in \mathcal{Z}(R)$ such that $\sigma(c) \neq c$. Then $(c-\sigma(c))^{2}$ is non-zero and belongs to the field $\mathcal{Z}(R)^{\sigma}$. Thus $c-\sigma(c)$ is invertible. Now let $\partial \in L_{1}$ and $x \in R$. Notice that

$$
\partial(x) c+\sigma(x) \partial(c)=\partial(x c)=\partial(c x)=\partial(c) x+\sigma(c) \partial(x) .
$$

In particular, we have

$$
\partial(x)=(c-\sigma(c))^{-1} \partial(c) x-\sigma(x)(c-\sigma(c))^{-1} \partial(c)=\partial_{b}(x),
$$

where $b=(c-\sigma(c))^{-1} \partial(c)$. Thus $L_{1}$ acts on $R$ via inner $\sigma$-derivations and in, particular, every mapping from $L$ is $\mathcal{Z}(R)^{\sigma}$-linear. We will prove that $\mathcal{Z}\left(R^{L_{0}}\right)^{\sigma}=\mathcal{Z}(R)^{\sigma}$. Similarly as above, it suffices to show that $\mathcal{Z}\left(R^{L_{0}}\right)^{\sigma} \subseteq R^{L_{1}}$. Take any $\partial=\partial_{b} \in L_{1}$, where $b=$ $(c-\sigma(c))^{-1} \partial(c)$ for some $c \in \mathcal{Z}(R)$. Since $L_{0}$ acts trivially on the centre of $R$, one obtains that $b \in R^{L_{0}}$. Now it is clear that $\partial_{b}$ acts trivially on $\mathcal{Z}\left(R^{L_{0}}\right)^{\sigma}$, and consequently $\mathcal{Z}\left(R^{L_{0}}\right)^{\sigma} \subseteq R^{L_{1}}$.

Consider skew group rings $R * G$ and $R^{L_{0}} * G$. Since both of $R$ and $R^{L_{0}}$ are $G$ simple, and $\sigma$ is outer on $R$ and $R^{L_{0}}$, the rings $R * G$ and $R^{L_{0}} * G$ are simple. Moreover it is clear that $\mathcal{Z}(R * G)=\mathcal{Z}(R)^{\sigma}$ and $\mathcal{Z}\left(R^{L_{0}} * G\right)=\mathcal{Z}\left(R^{L_{0}}\right)^{\sigma}$. Thus $R * G$ and $R^{L_{0}} * G$ are central simple $\mathcal{Z}(R)^{\sigma}$-algebras. Notice that the action of $L_{0}$ on $R$ can be extended to an action on $R * G$, via the formula $\delta(a+b \sigma)=\delta(a)+\delta(b) \sigma$. In that case $(R * G)^{L_{0}}=$ $R^{L_{0}} * G$ Again applying Lemma 2 we obtain that $L_{0}$ must act trivially on $R$ and the proof is complete.

COROLlary 5. Let a nilpotent Lie superalgebra $L=L_{0} \oplus L_{1}$ acts on a G-simple finite-dimensional $\mathbb{K}$-algebra $R$ with centre $\mathcal{Z}$, where char $\mathbb{K}=0$. If $R^{L} \subseteq \mathcal{Z}$ and $\left[L_{0}, L_{1}\right]=0$, then $\operatorname{dim}_{\mathcal{Z}^{G}} R \leq\left[\mathcal{Z}: \mathcal{Z}^{G}\right] \cdot 2^{\operatorname{dim}_{\kappa} L_{1}}$. Moreover, in this case $R$ satisfies the standard polynomial identity of degree $2 \cdot\left[\sqrt{2^{\operatorname{dim}_{\varangle} L_{1}}}\right]$.

Proof. By Proposition $4, L_{0}=0$. Thus $L$ is spanned by a family $\left\{\partial_{1}, \ldots, \partial_{n}\right\}$ of inner skew derivations such that $\partial_{j}^{2}=0$ and $\partial_{i} \partial_{j}+\partial_{j} \partial_{i}=0$. It is clear that every $\partial_{j}$ is $\mathcal{Z}^{G}$-linear. Let us consider a chain

$$
V_{0}=R \supseteq V_{1} \supseteq \cdots \supseteq V_{n}
$$

of subspaces of $R$, where $V_{j}=\operatorname{ker} \partial_{1} \cap \cdots \cap \operatorname{ker} \partial_{j}$ for $j=1, \ldots, n$. Then $V_{n} \subseteq R^{L} \subseteq \mathcal{Z}$ and $\partial_{j}$ maps $V_{j-1}$ into $V_{j}$. Moreover, it is clear that $\operatorname{dim}_{\mathcal{Z}^{G}} V_{j-1}=\operatorname{dim}_{\mathcal{Z}^{G}}\left(\operatorname{ker} \partial_{j} \cap V_{j-1}\right)+$ $\operatorname{dim}_{\mathcal{Z}^{G}} \partial_{j}\left(V_{j-1}\right) \leq 2 \cdot \operatorname{dim}_{\mathcal{Z}^{G}} V_{j}$. Thus

$$
\operatorname{dim}_{\mathcal{Z}^{G}} R \leq 2^{n} \cdot \operatorname{dim}_{\mathcal{Z}^{G}} V_{n} \leq\left[\mathcal{Z}: \mathcal{Z}^{G}\right] \cdot 2^{\operatorname{dim}_{\nVdash} L_{1}} .
$$

Since $R$ is $G$-simple, the algebra $R$ must be either simple or $R=I \oplus \sigma(I)$ for a minimal ideal $I$ of $R$. Then $I$ is certainly a simple algebra. The above inequality implies that 
$\operatorname{dim}_{\mathcal{Z}} R \leq 2^{\operatorname{dim}_{\varangle} L_{1}}$ in the first case, and $\operatorname{dim}_{\mathcal{Z}(I)} I \leq 2^{\operatorname{dim}_{\varangle} L_{1}}$ in the second case. The result follows now by the Amitsur-Levitzki theorem.

If $R$ is semiprime we let $Q=Q(R)$ to denote the symmetric Martindale quotient ring. Its centre, known as the extended centroid of $R$, we denote by $C$. The following properties of $Q$ in the case when $R$ is acted on by a Hopf algebra are proved in [3].

Lemma 6. Let $R$ be a semiprime $H$-module algebra such that the $H$-action on $R$ extends to an $H$-action on $Q$ and any non-zero $H$-stable ideal of $R$ contains non-trivial invariants. Then

(1) the ring $C^{H}=C \cap Q^{H}$ is von Neumann regular and selfinjective.

(2) If a non-empty subset $S \subseteq C^{H} \backslash\{0\}$ is closed under a multiplication, then the localization $Q_{S}$ of $Q$ at $S$ is semiprime and $\mathcal{Z}\left(Q_{S}\right)=C_{S}$.

(3) If $H$ acts finitely on $Q$ and $S=C^{H} \backslash M$, where $M$ is a maximal ideal of $C^{H}$, then the H-action on $Q$ extends to an $H$-action on $Q_{S}$ and $\left(Q^{H}\right)_{S}=\left(Q_{S}\right)^{H}$, $\left(C^{H}\right)_{S}=\left(C_{S}\right)^{H}=C_{S} \cap\left(Q_{S}\right)^{H}$ is a field contained in the centre of $Q_{S}$.

We can now prove the main result of the paper.

Proof of Theorem 1. Let $H=U(L) * G$. By ([2], Corollary 6) every $H$-invariant non-nilpotent subalgebra of $R$ contains non-zero invariants. Thus we can apply the results from [4]. Let $M$ be a maximal ideal of $C^{H}=C \cap Q^{H}$ and put $S=C^{H} \backslash M$. By the above lemma and [4] it follows that $\left(C_{S}\right)^{H}$ is a field and $Q_{S}$ is a finite dimensional, $G$-simple $\left(C_{S}\right)^{H}$-algebra. Using Corollary 5 we obtain that $Q_{M}$ satisfies the standard polynomial identity of degree $2 \cdot\left[\sqrt{2^{\operatorname{dim}_{\ll} L_{1}}}\right]$. Since it holds for any maximal ideal $M$ of $C^{H}$, the ring $Q$, and consequently $R$, satisfies the standard polynomial identity of degree $2 \cdot\left[\sqrt{2^{\operatorname{dim}_{\aleph} L_{1}}}\right]$.

3. Examples. In this section, we construct examples of actions of nilpotent Lie superalgebras with central invariants and with $\left[L_{0}, L_{1}\right] \neq 0$. We start with general properties of inner derivations and skew derivations of an algebra $R$ with an automorphism $\sigma$ of order 2. Then $R=R_{0} \oplus R_{1}$ is $\mathbb{Z}_{2}$-graded, where $R_{0}=\{x \in$ $R \mid \sigma(x)=x\}$ and $R_{1}=\{x \in R \mid \sigma(x)=-x\}$. For any inner derivation $\delta$ of $R$, the condition $\delta \sigma=\sigma \delta$ is equivalent to that $\delta$ is induced by some $a \in R_{0}$. To see that, we let $\delta$ be induced by $a=a_{0}+a_{1} \in R$. Then

$$
\delta(x)=a x-x a=\left(a_{0} x-x a_{0}\right)+\left(a_{1} x-x a_{1}\right) .
$$

This immediately implies that

$$
\delta(\sigma(x))=\left(a_{0} \sigma(x)-\sigma(x) a_{0}\right)+\left(a_{1} \sigma(x)-\sigma(x) a_{1}\right)
$$

and

$$
\sigma(\delta(x))=\left(a_{0} \sigma(x)-\sigma(x) a_{0}\right)-\left(a_{1} \sigma(x)-\sigma(x) a_{1}\right) .
$$

Since $\delta$ and $\sigma$ commute, the previous equations imply that $a_{1} \sigma(x)-\sigma(x) a_{1}=0$. Replacing $x$ by $\sigma(x)$ yields $a_{1} x-x a_{1}=0$. Equation (1) now becomes

$$
\delta(x)=a_{0} x-x a_{0}=\operatorname{ad}_{a_{0}}(x) .
$$


In the same manner we can show that for any inner skew derivation $\partial$ of $R$, the condition $\partial \sigma=-\sigma \partial$ is equivalent to that $\partial=\partial_{b}$ for some $b \in R_{1}$.

LEMMA 7. Let $R$ be an algebra over a field $\mathbb{K}$ of characteristic $\neq 2$ and $\sigma$ be a $\mathbb{K}$-linear automorphism of $R$ of order 2. Let $u \in R$ be invertible and $\sigma(u)=-u$. Let $\widetilde{R}$ be the $\mathbb{K}$-algebra $M_{2}(R)$, the $2 \times 2$ matrices over $R$. Then the map $\varphi: R \rightarrow \widetilde{R}$ given by

$$
\varphi(x)=\left(\begin{array}{cc}
x & 0 \\
0 & u^{-1} \sigma(x) u
\end{array}\right)
$$

is an injective homomorphism of algebras, satisfying $\tilde{\sigma} \varphi=\varphi \sigma$ (where $\tilde{\sigma}$ is a componentwise extension of $\sigma$ to $\widetilde{R}$ ).

If a Lie superalgebra $L=L_{0} \oplus L_{1}$ acts on $R$ by inner derivations and inner $\sigma$ derivations with $R^{L}=\mathbb{K}$, then L acts on $\widetilde{R}$ by inner derivations and inner $\widetilde{\sigma}$-derivations with

$$
\widetilde{R}^{L}=\left\{\left(\begin{array}{cc}
\alpha & \beta u \\
\gamma u^{-1} & \lambda
\end{array}\right) \in \widetilde{R} \mid \alpha, \beta, \gamma, \lambda \in \mathbb{K}\right\}
$$

Proof. Notice that

$$
(\widetilde{\sigma} \varphi)(x)=\widetilde{\sigma}\left(\left(\begin{array}{cc}
x & 0 \\
0 & u^{-1} \sigma(x) u
\end{array}\right)\right)=\left(\begin{array}{cc}
\sigma(x) & 0 \\
0 & u^{-1} x u
\end{array}\right)=(\varphi \sigma)(x) .
$$

In order to prove the second part, observe that for all inner derivation $\operatorname{ad}_{a} \in L_{0}$ and the inner skew derivation $\partial_{b} \in L_{1}$ of $R$ and for every matrix $\widetilde{x}=\left(\begin{array}{ll}x_{11} & x_{12} \\ x_{21} & x_{22}\end{array}\right) \in \widetilde{R}$ the following equations hold

$\operatorname{ad}_{\varphi(a)}(\widetilde{x})=\left(\begin{array}{cc}a & 0 \\ 0 & u^{-1} a u\end{array}\right) \cdot \tilde{x}-\tilde{x} \cdot\left(\begin{array}{cc}a & 0 \\ 0 & u^{-1} a u\end{array}\right)=\left(\begin{array}{cc}\operatorname{ad}_{a}\left(x_{11}\right) & \operatorname{ad}_{a}\left(x_{12} u^{-1}\right) u \\ u^{-1} \operatorname{ad}_{a}\left(u x_{21}\right) & u^{-1} \operatorname{ad}_{a}\left(u x_{22} u^{-1}\right) u\end{array}\right)$

and

$$
\begin{aligned}
\partial_{\varphi(b)}(\tilde{x}) & =\left(\begin{array}{cc}
b & 0 \\
0 & -u^{-1} b u
\end{array}\right) \cdot \tilde{x}-\tilde{\sigma}(\tilde{x}) \cdot\left(\begin{array}{cc}
b & 0 \\
0 & -u^{-1} b u
\end{array}\right) \\
& =\left(\begin{array}{cc}
\partial_{b}\left(x_{11}\right) & \partial_{b}\left(x_{12} u^{-1}\right) u \\
\sigma\left(u^{-1}\right) \partial_{b}\left(u x_{21}\right) & \sigma\left(u^{-1}\right) \partial_{b}\left(u x_{22} u^{-1}\right) u
\end{array}\right) .
\end{aligned}
$$

From the above equations it follows that $\widetilde{x} \in \widetilde{R}^{L}$ if and only if the elements $x_{11}, x_{12} u^{-1}$, $u x_{21}$ and $u x_{22} u^{-1}$ belong to $R^{L}$. Under the assumption that $R^{L}=\mathbb{K}$, we now easily obtain the assertion of the lemma.

We start our construction from the algebra $R=M_{2}(\mathbb{K})$ of $2 \times 2$ matrices over a field $\mathbb{K}$ of characteristic 0 . Let $\sigma$ be the inner automorphism of order 2 of $R$ induced 
by the diagonal matrix $\operatorname{diag}(1,-1)$ and let $\partial_{b_{1}}$ and $\partial_{b_{2}}$ be the inner $\sigma$-derivations of $R$ induced by

$$
b_{1}=\left(\begin{array}{ll}
0 & 1 \\
1 & 0
\end{array}\right) \in R_{1} \text { and } b_{2}=\left(\begin{array}{cc}
0 & 1 \\
-1 & 0
\end{array}\right) \in R_{1}
$$

respectively. It can be easily checked that

$$
b_{1}^{2}=-b_{2}^{2}=1 \text { and } b_{1} b_{2}+b_{2} b_{1}=0 .
$$

As a result, the skew derivations $\partial_{b_{1}}$ and $\partial_{b_{2}}$ span an Abelian Lie superalgebra $L=$ $L_{0} \oplus L_{1}$ where $L_{0}=0$ and $L_{1}=\operatorname{Span}_{\mathbb{}}\left\{\partial_{b_{1}}, \partial_{b_{2}}\right\}$. From the explicit formulas for $\partial_{b_{1}}$ and $\partial_{b_{2}}$

$$
\partial_{b_{1}}\left(\left(\begin{array}{ll}
x_{11} & x_{12} \\
x_{21} & x_{22}
\end{array}\right)\right)=\left(\begin{array}{ll}
x_{21}+x_{12} & x_{22}-x_{11} \\
x_{11}-x_{22} & x_{21}+x_{12}
\end{array}\right)
$$

and

$$
\partial_{b_{2}}\left(\left(\begin{array}{ll}
x_{11} & x_{12} \\
x_{21} & x_{22}
\end{array}\right)\right)=\left(\begin{array}{ll}
x_{21}-x_{12} & x_{22}-x_{11} \\
x_{22}-x_{11} & x_{21}-x_{12}
\end{array}\right)
$$

it follows immediately that $R^{L}=\mathbb{K}$.

Using Lemma 7, applied to the invertible element $u=b_{2}$, we have an embedding of $R$ into $\widetilde{R}=M_{2}(R)$, according to the formula

$$
\varphi(x)=\left(\begin{array}{cc}
x & 0 \\
0 & b_{2}^{-1} \sigma(x) b_{2}
\end{array}\right) .
$$

Put

$$
\widetilde{b_{1}}=\varphi\left(b_{1}\right)=\left(\begin{array}{cc}
b_{1} & 0 \\
0 & b_{1}
\end{array}\right) \in \widetilde{R}_{1} \text { and } \widetilde{b_{2}}=\varphi\left(b_{2}\right)=\left(\begin{array}{cc}
b_{2} & 0 \\
0 & -b_{2}
\end{array}\right) \in \widetilde{R}_{1}
$$

and consider the additional matrices

$$
\widetilde{b_{3}}=\left(\begin{array}{cc}
0 & b_{2} \\
-b_{2} & 0
\end{array}\right) \in \widetilde{R}_{1} \text { and } \widetilde{b_{4}}=\left(\begin{array}{cc}
0 & b_{2} \\
b_{2} & 0
\end{array}\right) \in \widetilde{R}_{1} .
$$

It is not hard to check that

$$
{\widetilde{b_{1}}}^{2}=-{\widetilde{b_{2}}}^{2}={\widetilde{b_{3}}}^{2}=-{\widetilde{b_{4}}}^{2}=1 \text { and } \widetilde{b}_{i} \widetilde{b}_{j}+\widetilde{b_{j}} \widetilde{b_{i}}=0
$$

for all $i \neq j$. As before, the inner skew derivations $\partial_{\widetilde{b_{1}}}, \partial_{\widetilde{b_{2}}}, \partial_{\widetilde{b_{3}}}$ and $\partial_{\widetilde{b_{4}}}$ span an Abelian Lie superalgebra $\widetilde{L}=\widetilde{L}_{0} \oplus \widetilde{L}_{1}$, where $\widetilde{L}_{0}=0$ and $\widetilde{L}_{1}=\operatorname{span}_{\mathbb{K}}\left\{\partial_{\widetilde{b_{1}}}, \partial_{\widetilde{b}_{2}}, \partial_{\widetilde{b_{3}}}, \partial_{\widetilde{b_{4}}}\right\}$. Lemma 7 says that the subalgebra of invariants $\widetilde{R}^{L}$ under the action of $L$ consists of all matrices of the form $\left(\begin{array}{cc}\alpha & \beta b_{2} \\ \gamma b_{2} & \lambda\end{array}\right)$ where $\alpha, \beta, \gamma, \lambda \in \mathbb{K}$. Furthermore, a simple calculation shows that

$$
\partial_{\tilde{b_{3}}}\left(\left(\begin{array}{cc}
\alpha & \beta b_{2} \\
\gamma b_{2} & \lambda
\end{array}\right)\right)=\left(\begin{array}{cc}
\beta-\gamma & (\lambda-\alpha) b_{2} \\
(\lambda-\alpha) b_{2} & \beta-\gamma
\end{array}\right)
$$


and

$$
\partial_{\tilde{b}_{4}}\left(\left(\begin{array}{cc}
\alpha & \beta b_{2} \\
\gamma b_{2} & \lambda
\end{array}\right)\right)=\left(\begin{array}{cc}
-\beta-\gamma & (\lambda-\alpha) b_{2} \\
(\alpha-\lambda) b_{2} & -\beta-\gamma
\end{array}\right)
$$

This immediately implies that $\widetilde{R}^{L}=\mathbb{K}$.

Applying Lemma 7 for the invertible element $u=\widetilde{b_{4}}$ we have the next embedding of $\widetilde{R}$ into the algebra $R=M_{2}(\widetilde{R})$, the $2 \times 2$ matrices over $\widetilde{R}$ according to the formula

$$
\varphi(\widetilde{x})=\left(\begin{array}{cc}
\widetilde{x} & 0 \\
0 & {\widetilde{b_{4}}}^{-1} \tilde{\sigma}(\widetilde{x}) \tilde{b}_{4}
\end{array}\right)
$$

Put

$$
B_{i}=\varphi\left(\widetilde{b}_{i}\right)=\left(\begin{array}{cc}
\widetilde{b}_{i} & 0 \\
0 & \widetilde{b_{i}}
\end{array}\right) \in \boldsymbol{R}_{1} \text { and } B_{4}=\varphi\left(\widetilde{b}_{4}\right)=\left(\begin{array}{cc}
\widetilde{b}_{4} & 0 \\
0 & -\widetilde{b_{4}}
\end{array}\right) \in \boldsymbol{R}_{1}
$$

for $i=1,2,3$ and consider the additional matrices

- $A_{1}=\left(\begin{array}{cc}0 & \widetilde{a_{1}} \\ -\widetilde{a}_{1} & 0\end{array}\right) \in \boldsymbol{R}_{0}$ and $C_{1}=\left(\begin{array}{cc}0 & \widetilde{a_{1}} \\ 0 & 0\end{array}\right) \in \boldsymbol{R}_{0}$, where $\widetilde{a_{1}}=\left(\begin{array}{cc}1 & 1 \\ -1 & -1\end{array}\right) \in \widetilde{R}_{0}$,

- $A_{2}=\left(\begin{array}{cc}0 & \widetilde{a_{2}}+1 \\ -\widetilde{a}_{2}+1 & 0\end{array}\right) \in \boldsymbol{R}_{0}$ and $C_{2}=\left(\begin{array}{cc}0 & \widetilde{a_{2}}+1 \\ 0 & 0\end{array}\right) \in \boldsymbol{R}_{0}$, where $\widetilde{a_{2}}=\left(\begin{array}{cc}0 & b_{1} b_{2} \\ b_{1} b_{2} & 0\end{array}\right) \in \widetilde{R}_{0}$,

- $A_{3}=\left(\begin{array}{cc}\widetilde{a_{3}}-\widetilde{a_{1}} & 0 \\ 0 & \widetilde{a_{3}}+\widetilde{a_{1}}\end{array}\right) \in \boldsymbol{R}_{0}$, where $\widetilde{a_{3}}=\left(\begin{array}{cc}b_{1} b_{2} & b_{1} b_{2} \\ -b_{1} b_{2} & -b_{1} b_{2}\end{array}\right) \in \widetilde{R}_{0}$,

- $B_{5}=\left(\begin{array}{cc}0 & \widetilde{d_{5}} \\ \widetilde{b_{5}} & 0\end{array}\right) \in \boldsymbol{R}_{1}, B_{6}=\left(\begin{array}{cc}0 & \widetilde{b_{4}} \\ -\widetilde{b_{4}} & 0\end{array}\right) \in \boldsymbol{R}_{1}$ and $B_{7}=\left(\begin{array}{cc}0 & \widetilde{b_{4}} \\ \widetilde{b_{4}} & 0\end{array}\right) \in \boldsymbol{R}_{1}$, where

$\tilde{d}_{5}=\left(\begin{array}{cc}b_{1}+b_{2} & b_{1}+b_{2} \\ -b_{1}-b_{2} & -b_{1}-b_{2}\end{array}\right), \tilde{b}_{5}=\left(\begin{array}{cc}-b_{1}+b_{2} & -b_{1}+b_{2} \\ b_{1}-b_{2} & b_{1}-b_{2}\end{array}\right) \in \widetilde{R}_{1}$,

- $D_{5}=\left(\begin{array}{cc}0 & \widetilde{d}_{5} \\ 0 & 0\end{array}\right)+B_{7} \in \boldsymbol{R}_{1}$ and $D_{6}=\left(\begin{array}{cc}0 & \widetilde{b_{4}} \\ 0 & 0\end{array}\right) \in \boldsymbol{R}_{1}$.

Notice that if $N_{0}=\operatorname{span}_{\mathbb{K}}\left\{\operatorname{ad}_{C_{1}}, \operatorname{ad}_{C_{2}}, \operatorname{ad}_{A_{3}}\right\}$ and $N_{1}=\operatorname{span}_{\mathbb{K}}\left\{\partial_{B_{1}}, \partial_{B_{2}}, \partial_{B_{3}}, \partial_{B_{4}}\right.$, $\partial_{D_{5}}, \partial_{D_{6}}$, then $\boldsymbol{N}=\boldsymbol{N}_{0} \oplus \boldsymbol{N}_{1}$ is a nine-dimensional Lie superalgebra of nilpotency class 4 (see Table 1). Lemma 7 asserts that the subalgebra of invariants $\boldsymbol{R}^{\widetilde{L}}$ under the action of $\widetilde{L}$ consists of all matrices of the form $\left(\begin{array}{cc}\alpha & \beta \widetilde{b_{4}} \\ \gamma & \tilde{b}_{4}\end{array}\right)$, where $\alpha, \beta, \gamma, \lambda \in \mathbb{K}$. Moreover,

$$
\begin{aligned}
& \partial_{D_{5}}\left(\left(\begin{array}{cc}
\alpha & \beta \widetilde{b_{4}} \\
\gamma \widetilde{b_{4}} & \lambda
\end{array}\right)\right)=\left(\begin{array}{cc}
\gamma \widetilde{d}_{5}{\widetilde{b_{4}}}-\beta-\gamma & (\lambda-\alpha)\left(\widetilde{b_{4}}+\widetilde{d}_{5}\right) \\
(\alpha-\lambda) \tilde{b}_{4} & \gamma \widetilde{b}_{4}{\tilde{d_{5}}}-\beta-\gamma
\end{array}\right) \\
& =\left(\begin{array}{cc}
\gamma\left(\widetilde{a_{3}}-\tilde{a_{1}}\right)-\beta-\gamma & (\lambda-\alpha)\left(\widetilde{b_{4}}+\tilde{d}_{5}\right) \\
(\alpha-\lambda) \widetilde{b_{4}} & \gamma\left(\widetilde{a_{3}}+\widetilde{a_{1}}\right)-\beta-\gamma
\end{array}\right) \text {. }
\end{aligned}
$$

As a result we obtain that $\boldsymbol{R}^{N}=\mathbb{K}$. 
Table 1. Operation table of $N$

\begin{tabular}{cccccccccc}
\hline$[\cdot, \cdot]$ & $\operatorname{ad}_{C_{1}}$ & $\mathrm{ad}_{C_{2}}$ & $\mathrm{ad}_{A_{3}}$ & $\partial_{B_{1}}$ & $\partial_{B_{2}}$ & $\partial_{B_{3}}$ & $\partial_{B_{4}}$ & $\partial_{D_{5}}$ & $\partial_{D_{6}}$ \\
\hline $\operatorname{ad}_{C_{1}}$ & 0 & 0 & 0 & 0 & $-2 \partial_{D_{6}}$ & $2 \partial_{D_{6}}$ & 0 & $\partial_{B_{2}+B_{3}}$ & 0 \\
$\mathrm{ad}_{C_{2}}$ & 0 & 0 & 0 & $2 \partial_{D_{6}}$ & 0 & 0 & $2 \partial_{D_{6}}$ & $-\partial_{B_{1}-B_{4}}$ & 0 \\
$\mathrm{ad}_{A_{3}}$ & 0 & 0 & 0 & $-2 \partial_{B_{2}+B_{3}}$ & $-2 \partial_{B_{1}-B_{4}}$ & $2 \partial_{B_{1}-B_{4}}$ & $-2 \partial_{B_{2}+B_{3}}$ & 0 & 0 \\
$\partial_{B_{1}}$ & 0 & $-2 \partial_{D_{6}}$ & $2 \partial_{B_{2}+B_{3}}$ & 0 & 0 & 0 & 0 & $2 \mathrm{ad}_{C_{1}}$ & 0 \\
$\partial_{B_{2}}$ & $2 \partial_{D_{6}}$ & 0 & $2 \partial_{B_{1}-B_{4}}$ & 0 & 0 & 0 & 0 & $-2 \mathrm{ad}_{C_{2}}$ & 0 \\
$\partial_{B_{3}}$ & $-2 \partial_{D_{6}}$ & 0 & $-2 \partial_{B_{1}-B_{4}}$ & 0 & 0 & 0 & 0 & $2 \mathrm{ad}_{C_{2}}$ & 0 \\
$\partial_{B_{4}}$ & 0 & $-2 \partial_{D_{6}}$ & $2 \partial_{B_{2}+B_{3}}$ & 0 & 0 & 0 & 0 & $2 \mathrm{ad}_{C_{1}}$ & 0 \\
$\partial_{D_{5}}$ & $-\partial_{B_{2}+B_{3}}$ & $\partial_{B_{1}-B_{4}}$ & 0 & $2 \mathrm{ad}_{C_{1}}$ & $-2 \mathrm{ad}_{C_{2}}$ & $2 \mathrm{ad}_{C_{2}}$ & $2 \mathrm{ad}_{C_{1}}$ & $2 \mathrm{ad}_{A_{3}}$ & 0 \\
$\partial_{D_{6}}$ & 0 & 0 & 0 & 0 & 0 & 0 & 0 & 0 & 0 \\
\hline
\end{tabular}

Table 2. Operation table of $\boldsymbol{L}$

\begin{tabular}{ccccccccccc}
\hline$[\cdot, \cdot]$ & $\mathrm{ad}_{A_{1}}$ & $\mathrm{ad}_{A_{2}}$ & $\mathrm{ad}_{A_{3}}$ & $\partial_{B_{1}}$ & $\partial_{B_{2}}$ & $\partial_{B_{3}}$ & $\partial_{B_{4}}$ & $\partial_{B_{5}}$ & $\partial_{B_{6}}$ & $\partial_{B_{7}}$ \\
\hline $\mathrm{ad}_{A_{1}}$ & 0 & $-2 \mathrm{ad}_{A_{3}}$ & 0 & 0 & $-2 \partial_{B_{6}}$ & $2 \partial_{B_{6}}$ & 0 & 0 & $-2 \partial_{B_{2}+B_{3}}$ & 0 \\
$\mathrm{ad}_{A_{2}}$ & $2 \mathrm{ad}_{A_{3}}$ & 0 & 0 & $2 \partial_{B_{6}}$ & 0 & 0 & $2 \partial_{B_{6}}$ & 0 & $2 \partial_{B_{1}-B_{4}}$ & 0 \\
$\mathrm{ad}_{A_{3}}$ & 0 & 0 & 0 & $-2 \partial_{B_{2}+B_{3}}$ & $-2 \partial_{B_{1}-B_{4}}$ & $2 \partial_{B_{1}-B_{4}}$ & $-2 \partial_{B_{2}+B_{3}}$ & 0 & 0 & 0 \\
$\partial_{B_{1}}$ & 0 & $-2 \partial_{B_{6}}$ & $2 \partial_{B_{2}+B_{3}}$ & 0 & 0 & 0 & 0 & $2 \mathrm{ad}_{A_{1}}$ & 0 & 0 \\
$\partial_{B_{2}}$ & $2 \partial_{B_{6}}$ & 0 & $2 \partial_{B_{1}-B_{4}}$ & 0 & 0 & 0 & 0 & $-2 \mathrm{ad}_{A_{2}}$ & 0 & 0 \\
$\partial_{B_{3}}$ & $-2 \partial_{B_{6}}$ & 0 & $-2 \partial_{B_{1}-B_{4}}$ & 0 & 0 & 0 & 0 & $2 \mathrm{ad}_{A_{2}}$ & 0 & 0 \\
$\partial_{B_{4}}$ & 0 & $-2 \partial_{B_{6}}$ & $2 \partial_{B_{2}+B_{3}}$ & 0 & 0 & 0 & 0 & $2 \mathrm{ad}_{A_{1}}$ & 0 & 0 \\
$\partial_{B_{5}}$ & 0 & 0 & 0 & $2 \mathrm{ad}_{A_{1}}$ & $-2 \mathrm{ad}_{A_{2}}$ & $2 \mathrm{ad}_{A_{2}}$ & $2 \mathrm{ad}_{A_{1}}$ & 0 & $-2 \mathrm{ad}_{A_{3}}$ & 0 \\
$\partial_{B_{6}}$ & $2 \partial_{B_{2}+B_{3}}$ & $-2 \partial_{B_{1}-B_{4}}$ & 0 & 0 & 0 & 0 & 0 & $-2 \mathrm{ad}_{A_{3}}$ & 0 & 0 \\
$\partial_{B_{7}}$ & 0 & 0 & 0 & 0 & 0 & 0 & 0 & 0 & 0 & 0 \\
\hline
\end{tabular}

Notice also that if $\boldsymbol{M}_{0}=\operatorname{span}_{\mathbb{K}}\left\{\operatorname{ad}_{A_{1}}, \operatorname{ad}_{A_{2}}, \operatorname{ad}_{A_{3}}\right\}$ and $\boldsymbol{M}_{1}=\operatorname{span}_{\mathbb{K}}\left\{\partial_{B_{1}}, \partial_{B_{2}}, \partial_{B_{3}}\right.$, $\left.\partial_{B_{4}}, \partial_{B_{5}+B_{7}}, \partial_{B_{6}}\right\}$, then $\boldsymbol{M}=\boldsymbol{M}_{0} \oplus \boldsymbol{M}_{1}$ is a nilpotent Lie superalgebra of nilpotency class 6 (see Table 2). We have

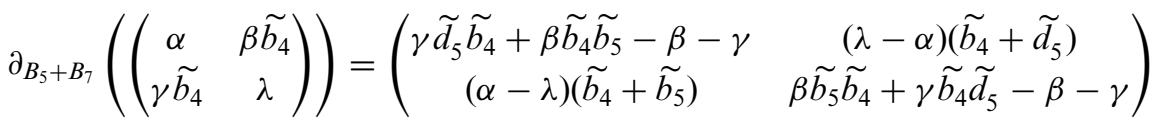

$$
\begin{aligned}
& =\left(\begin{array}{cc}
(\gamma-\beta)\left(\widetilde{a_{3}}-\tilde{a}_{1}\right)-\beta-\gamma & (\lambda-\alpha)\left(\widetilde{b_{4}}+\tilde{d}_{5}\right) \\
(\alpha-\lambda)\left(\widetilde{b_{4}}+\tilde{b}_{5}\right) & (\gamma-\beta)\left(\widetilde{a_{3}}+\widetilde{a_{1}}\right)-\beta-\gamma
\end{array}\right) \text {. }
\end{aligned}
$$

This implies immediately that $\boldsymbol{R}^{\boldsymbol{M}}=\mathbb{K}$.

Finally, observe also that $\boldsymbol{M}$ is an subalgebra of a nilpotent Lie superalgebra $\boldsymbol{L}=\boldsymbol{L}_{0} \oplus \boldsymbol{L}_{1}$ of nilpotency class 6, where $\boldsymbol{L}_{0}=\left[\boldsymbol{L}_{1}, \boldsymbol{L}_{1}\right]=\operatorname{span}_{\mathbb{K}}\left\{\operatorname{ad}_{A_{1}}, \operatorname{ad}_{A_{2}}, \operatorname{ad}_{A_{3}}\right\}$ and $\boldsymbol{L}_{1}=\operatorname{span}_{\mathbb{K}}\left\{\partial_{B_{1}}, \partial_{B_{2}}, \partial_{B_{3}}, \partial_{B_{4}}, \partial_{B_{5}}, \partial_{B_{6}}, \partial_{B_{7}}\right\}$ (see Table 2). Obviously, $\boldsymbol{R}^{\boldsymbol{L}}=\mathbb{K}$. Starting with the algebra $\boldsymbol{R}$, the invertible element $u=B_{7}$ and the Lie superalgebra $\boldsymbol{L}$, and again applying the above procedure, we can produce successive examples.

ACKNOWLEDGEMENTS. The first author's research was supported in part by KBN Grant No. 1 P03A 03227 and by the grant S/WI/3/2009 of Bialystok University of Technology.

\section{REFERENCES}

1. J. Bergen and P. Grzeszczuk, Invariants of Lie superalgebras acting on associative rings, Israel J. Math. 94 (1996), 403-428. 
2. J. Bergen and P. Grzeszczuk, Invariants of Lie color algebras acting on graded algebras, Colloq. Math. 83 (2000), 107-124.

3. P. Grzeszczuk and M. Hryniewicka, Polynomial identities of algebras with actions of pointed Hopf algebras, J. Algebra 278 (2004), 684-703.

4. P. Grzeszczuk and M. Hryniewicka, Actions of pointed Hopf algebras with reduced invariants, Proc. Amer. Math. Soc. 135 (2007), 2381-2389.

5. N. Jacobson, Structure of rings, vol. 37 (American Mathematical Society Colloquium Publications, Providence, RI, 1964). 